Nonlinear Processes in Geophysics (2002) 9: 79-86

Nonlinear Processes

in Geophysics

(C)European Geophysical Society 2002

\title{
Dynamics of nonlinear resonant slow MHD waves in twisted flux tubes
}

\author{
R. Erdélyi ${ }^{1}$ and I. Ballai ${ }^{2}$ \\ ${ }^{1}$ Space \& Atmosphere Research Center (SPARC), Dept. of Applied Mathematics, University of Sheffield, Hicks Building, \\ Hounsfield Road, Sheffield, S3 7RH, UK \\ ${ }^{2}$ School of Mathematics and Statistics, University of St Andrews, St Andrews, Fife, KY16 9SS, UK
}

Received: 12 June 2001 - Revised: 16 October 2001 - Accepted: 21 December 2001

\begin{abstract}
Nonlinear resonant magnetohydrodynamic (MHD) waves are studied in weakly dissipative isotropic plasmas in cylindrical geometry. This geometry is suitable and is needed when one intends to study resonant MHD waves in magnetic flux tubes (e.g. for sunspots, coronal loops, solar plumes, solar wind, the magnetosphere, etc.)

The resonant behaviour of slow MHD waves is confined in a narrow dissipative layer. Using the method of simplified matched asymptotic expansions inside and outside of the narrow dissipative layer, we generalise the so-called connection formulae obtained in linear MHD for the Eulerian perturbation of the total pressure and for the normal component of the velocity. These connection formulae for resonant MHD waves across the dissipative layer play a similar role as the well-known Rankine-Hugoniot relations connecting solutions at both sides of MHD shock waves.

The key results are the nonlinear connection formulae found in dissipative cylindrical MHD which are an important extension of their counterparts obtained in linear ideal MHD (Sakurai et al., 1991), linear dissipative MHD (Goossens et al., 1995; Erdélyi, 1997) and in nonlinear dissipative MHD derived in slab geometry (Ruderman et al., 1997).

These generalised connection formulae enable us to connect solutions obtained at both sides of the dissipative layer without solving the MHD equations in the dissipative layer possibly saving a considerable amount of CPU-time when solving the full nonlinear resonant MHD problem.
\end{abstract}

\section{Introduction}

One of the most interesting processes in the solar atmosphere is the complicated interaction of the plasma motions with magnetic fields. The solar atmosphere is a highly nonuniform plasma and, as a consequence, it is a natural medium for resonant MHD waves.

Correspondence to: R. Erdélyi (robertus@sheffield.ac.uk)
The dynamics of MHD waves is usually described in the framework of dissipative MHD. An important property of the governing wave equations is the smallness of the coefficients of the dissipative terms with respect to those of the ideal ones. On the other hand, dissipative terms contain the highest order derivatives, which means dissipation occurs only where strong gradients are present, or where the propagating wave develops small spatial scales across the equilibrium field. In a homogeneous plasma, the only transverse spatial scale is the wavelength in the direction normal to the field. Therefore, in order to have an efficient dissipation, the ratio between the perpendicular and parallel wavelengths must be very small. For this reason, damping of MHD waves in homogeneous plasma is not a viable heating mechanism. On the other hand, space missions of the past decade made it clear that space (in particular solar) plasma is strongly inhomogeneous. In such plasmas, the dissipative damping of MHD waves can be efficient, since the interaction of the propagating disturbance with the ambient background provides new ways of creating small transverse scales.

A direct consequence of the inhomogeneity is the possibility of the resonant transfer of energy between the plasma and driven waves. The absorbed energy from the driven waves can be converted into heat if the plasma is dissipative. Resonant absorption is one of the most popular processes to explain the anomalous behaviour of the temperature in the solar corona, i.e. the heating of the coronal plasma. The solar corona is a very hot tenuous plasma at a typical temperature of $1-3 \times 10^{6} \mathrm{~K}$, much higher than the underlying layers, (the transition region, the chromosphere and the photosphere) so nonthermal energy must be transported into the corona and be dissipated there. The high temperature coronal plasma mainly radiates in a soft $\mathrm{X}$-ray range corresponding to typical wavelengths of the order of $10-100 \AA$. This radiation is not homogeneous neither in space nor in time, with a wide range of spatial and time scales. High resolution observations have now given an image of the solar corona as a rapidly evolving dynamic plasma where energetic phenomena occur mainly on very "small" scales. 
The main problem in the understanding of coronal heating is to perform sufficiently high resolution observations (i.e. observations at typical wavelengths of the order of the dissipative length scales), capable of shedding light on the physical dissipative processes at work in the solar corona. As a consequence, no realistic models, even very simplified, have been developed so far, while a number of conceptual models derived from the available data try to roughly describe the small scale dynamics and to derive all the possible consequences on the mean dynamics in order to fit the observational large scale constraints (e.g. Litwin and Rosner, 1998, or more references in Bray et al., 1991; Golub and Pasachoff, 1997).

The physical background of resonant absorption is based on the existence of continuous spectra for slow MHD and Alfvén waves in inhomogeneous plasmas (Belien et al., 1997). Resonant absorption occurs whenever the frequency of a laterally driven oscillation matches the local slow and/or Alfvén frequency of the plasma and resonant field lines are created which transfer energy from the surface disturbance to its environment.

In ideal plasmas resonant waves are confined to an individual magnetic surface which cannot interact with its neighbors. Since we suppose that the driven external mode can exist for an infinitely long period, this energy accumulation results in infinite wave amplitude at the resonant position. But one of the basic characteristics of the solar plasma is that it is dissipative. Dissipative effects cause coupling of one resonant magnetic surface to neighboring magnetic surfaces and the disturbance provoked at the resonant surface is transmitted to neighboring field lines. We can define a "resonant layer" by considering a region where the disturbances do not become out of phase relative to the driven oscillations. For large values of the viscous and magnetic Reynolds numbers (just like in the solar atmosphere), this coupling is weak and the local resonant slow and Alfvén oscillations are characterized by steep gradients across the resonant magnetic field lines. In this case, the energy of the external oscillations is dissipated and it can be converted into heat. The total Reynolds number, which measures the magnitude of the dissipative effects, is defined by

$\frac{1}{R}=\frac{1}{R_{v}}+\frac{1}{R_{m}}$,

where $R_{v}$ and $R_{m}$ are the viscous and magnetic Reynolds numbers, respectively.

Ionson (1978) pointed out first that resonant absorption can be a viable candidate for coronal heating. Since then, this process became one of the most popular mechanisms for explaining the anomalous behaviour of the coronal temperature (see, e.g. Kuperus et al., 1981; Davila, 1987; Narain and Ulmschneider, 1990; Hollweg, 1991; Goossens, 1991; Narain and Ulmschneider, 1996, and references therein). The same mechanism may be used to explain the observed energy loss of $p$-modes in the vicinity of sunspots (see, e.g. Hollweg, 1988; Lou, 1990; Goossens and Poedts, 1992; Goossens and Hollweg, 1993; Erdélyi and Goossens, 1994).
A very elegant way to study resonant absorption in the vicinity of the resonant singularity is to use the concept of the so-called connection formulae introduced first by Sakurai et al. (1991) in ideal plasmas and by Goossens et al. (1995) and Erdélyi (1997) in dissipative plasmas. This approach is based on the idea that the thin dissipative layer acts as a surface of discontinuity when solving the MHD equations. At both sides of this surface of discontinuity the plasma motion is governed by the ideal MHD equations. The solution of the dissipative MHD equations inside the dissipative layer is used then to obtain the connection formulae which provide boundary conditions at the surface of discontinuity.

In the nonlinear description of resonant absorption we use some characteristic quantities, such as the characteristic scale of inhomogeneity, $l_{i n h}$, the characteristic thickness of the dissipative layer, $l_{d i s}$, and the dimensionless amplitude of perturbations $\epsilon$ far away from the dissipative layer. Under the plausible assumption that the characteristic scale of the perturbations along the dissipative layer is of the order of $l_{i n h}$, linear theory predicts that the perturbations of the large variables are of the order of $\epsilon l_{i n h} / l_{d i s}$ inside the dissipative layer (see, e.g. Goossens et al., 1995). Since the ratio $l_{i n h} / l_{d i s}$ is proportional to $R^{1 / 3}$, the amplitudes of the so-called large variables (for definition, see e.g. Goossens et al., 1995) can be very large inside the dissipative layer even when they are small outside. Note that the present analysis is valid for perturbations with characteristic wave numbers along the resonant dissipative layer for which

$\epsilon^{-1} \gg k l_{\text {inh }} \gg \epsilon$.

As a result, nonlinear theory can become important inside the dissipative layer.

Nonlinearity in the dissipative layer was first taken into account in the theory of resonant absorption by Ruderman et al. (1997a) where they studied the nonlinear evolution of slow resonant MHD waves considering Cartesian geometry and isotropic dissipative effects. Later, this theory was extended for anisotropic plasmas (e.g. solar corona) by Ballai et al. (1998b) where an anisotropic viscosity and fieldaligned thermal conductivity played the role of dissipative effects. These theories were applied to study the resonant absorption of sound and fast magneto-acoustic waves in solar structures (see, e.g. Ruderman et al., 1997b; Ballai et al., 1998a; Erdélyi and Ballai, 1999; Erdélyi et al., 2001). One of the main results of these studies was that in contrast to the linear theory, the coefficient of wave energy absorption is dependent on the particular type of dissipation. They have also found that the general tendency of nonlinearity is to decrease the absolute value of the coefficient of wave energy absorption when the wavelength of the incoming wave is much larger than the characteristic scale of inhomogeneity and nonlinearity is considered weak. This situation is changed in the limit of strong nonlinearity, as pointed out by Ruderman (2000), when this tendency does not persist anymore, at least for intermediate wavelengths. In the case of long wavelength approximation, nonlinearity again 
decreases the net coefficient of energy absorption and the difference relative to the results found by means of linear theory is about $20 \%$.

The goal of the present paper is to investigate how nonlinearity effects the dynamics of the resonant slow MHD waves in twisted magnetic flux tubes and to investigate the effect of the magnetic twisting on the connection formulae. We recall here a few recent studies on MHD wave dynamics when magnetic twist has been incorporated (e.g. Sakai et al., 2001; de Bruyne et al., 1996); however, they do not focus on the resonant aspect which is the key ingredient in our paper. The paper is organized as follows. In the next section, we introduce the basic equations and the equilibrium state. In order to obtain an equation that contains the effects of nonlinearity and dissipation, a scaling law is introduced in this section. In Sect. 3, we derive the governing equations for wave dynamics outside and inside the dissipative layer. Since the resonant surface acts like a singular surface, the connection formulae are calculated by means of the simplified method of matched asymptotic expansions. The generation of a mean flow due to resonant absorption of wave momentum is studied in Sect. 4. Finally, we summarize and discuss our results.

\section{Physical model}

The solar corona consists of myriads of loops. They can be up to $700000 \mathrm{~km}$ long with a radius between 1000 and $10000 \mathrm{~km}$ and longer loops having larger radii. Hence, the model of a cylindrical plasma column considered in the present paper is a good approximation for coronal loops.

The study of twisted tubes can be important in the context of solar physics. Many observational studies have revealed that the solar magnetic field is twisted. This is manifested in various kinds of observations, such as the morphology of $H_{\alpha}$ structures, the morphology of filaments and coronal loops and the signs of current helicity derived from vector magnetograms. (For more details see the review books, e.g. Bray et al., 1991; Golub and Pasachoff, 1997).

We consider a cylindrical plasma column described by cylindrical coordinates, $(r, \varphi, z)$, with longitudinal and helical equilibrium magnetic field. We suppose all equilibrium quantities depend on the radial variable, $r$, only. Gravity is ignored at the present stage.

\section{Nonlinear MHD equations}

We use the full set of nonlinear visco-resistive MHD equations

$$
\begin{aligned}
\frac{D \bar{\rho}}{D t} & +\bar{\rho} \nabla \cdot \boldsymbol{v}=0, \quad \nabla \cdot \boldsymbol{B}=0 \\
\frac{D \boldsymbol{v}}{D t} & =-\frac{\nabla \bar{p}}{\bar{\rho}}+\frac{1}{\mu \bar{\rho}}[(\nabla \times \boldsymbol{B}) \times \boldsymbol{B}]+ \\
& +\bar{v}\left(\nabla^{2} \boldsymbol{v}+\frac{1}{3} \nabla(\nabla \cdot \boldsymbol{v})\right)
\end{aligned}
$$

$$
\begin{aligned}
& \frac{\partial \boldsymbol{B}}{\partial t}=\nabla \times(\boldsymbol{v} \times \boldsymbol{B})+\bar{\eta} \nabla^{2} \boldsymbol{B}, \\
& \frac{D}{D t}\left(\frac{\bar{p}}{\bar{\rho}^{\gamma}}\right)=0, \quad D / D t=\partial / \partial t+\boldsymbol{v} \cdot \nabla .
\end{aligned}
$$

Here, $\boldsymbol{v}$ and $\boldsymbol{B}$ are the velocity and magnetic induction vectors, $\bar{p}$ and $\bar{\rho}$ are the pressure and density, $\bar{v}, \bar{\eta}$ and $\gamma$ denote the coefficient of kinematic shear viscosity, the magnetic diffusion and the adiabatic index, respectively. The perturbations of the magnetic field and velocity are denoted by $\boldsymbol{b}=\left(b_{r}, b_{\varphi}, b_{z}\right)$ and $\boldsymbol{v}=(u, v, w)$. In spite of the presence of dissipation we use the adiabatic equation as an approximation of the energy equation. Ballai et al. (2000) have shown that the dissipation due to viscosity and finite electrical conductivity present in the energy equation does not lead to a significant change in the behaviour of nonlinear slow resonant MHD waves in a driven problem. This property is based on the fact that the amplitudes of the nonlinear terms in the energy equation are larger than the amplitudes of the dissipative terms. This result is not applicable to nonlinear resonant Alfvén waves since in that case the two amplitudes may be of the same order.

We consider finite perturbations of the form

$\bar{f}=f_{0}(r)+f(r, \varphi, z, t)$,

where $f_{0}$ is the equilibrium value of a variable and $f$ is its Eulerian perturbation.

The equilibrium variables satisfy the radial force balance

$\frac{d}{d r}\left(p_{0}+\frac{B_{0}^{2}}{2 \mu}\right)=-\frac{B_{0 \varphi}^{2}}{\mu r}$,

where $B_{0}=\left(B_{0 \varphi}^{2}+B_{0 z}^{2}\right)^{1 / 2}$. With these variables, we can define the total pressure perturbation as

$P=p+\frac{\boldsymbol{B}^{2}}{2 \mu}=p+\frac{\boldsymbol{B}_{0} \cdot \boldsymbol{b}}{\mu}+\frac{\boldsymbol{b}^{2}}{2 \mu}$.

In linear theory all physical variables oscillate with the same real frequency, $\omega$ and they can be Fourier-analysed. However in nonlinear theory, this procedure cannot be applied since the oscillations are not in phase anymore; their propagation speed depends on the amplitude of the wave. To be as close as possible to the linear theory, we suppose that the oscillations are plane periodic propagating waves with permanent shape and we take all the variables to be dependent on the combination $\theta=m \varphi+k z-\omega t$ of the independent variables $\varphi, z$ and $\mathrm{t}$ rather than on $\varphi, z$ and $t$ separately. Here, $m$ and $k$ are the azimuthal and longitudinal wave numbers. The square of the Alfvén and sound speeds, and the Alfvén and the cusp frequencies are defined as

$v_{A}^{2}=\frac{B_{0}^{2}}{\mu \rho_{0}}, \quad c_{S}^{2}=\frac{\gamma p_{0}}{\rho_{0}}, \quad \omega_{A}^{2}=\frac{f_{B}^{2}}{\mu \rho_{0}}, \quad \omega_{T}^{2}=\frac{\omega_{A}^{2} c_{S}^{2}}{v_{A}^{2}+c_{S}^{2}}$,

where

$f_{B}=\frac{m}{r} B_{0 \varphi}+k B_{0 z}, \quad g_{B}=\frac{m}{r} B_{0 z}-k B_{0 \varphi}$. 
Let us introduce the parallel and perpendicular components of the velocity and magnetic field relative to the equilibrium magnetic field as

$$
\begin{aligned}
& \left(v_{\|}, b_{\|}\right)=(\boldsymbol{v}, \boldsymbol{b}) \cdot \frac{\boldsymbol{B}_{0}}{B_{0}}, \\
& \quad\left(v_{\perp}, b_{\perp}\right)=\frac{1}{B_{0}}\left[\left(v, b_{\varphi}\right) B_{0 z}-\left(w, b_{z}\right) B_{0 \varphi}\right] .
\end{aligned}
$$

The resonant wave properties are studied near and far from the resonant surface in the next section.

\section{The wave dynamics in the dissipative layer}

The mathematical procedure applied to derive the equation that describes the resonant slow wave motion in the dissipative layer is based on the simplified method of matched asymptotic expansions developed by Ruderman et al. (1997a) and Ballai et al. (1998b) from a more general description of the method (Nayfeh, 1981).

The simplified version of the method of matched asymptotic expansion is based on very simple ideas. Since we are dealing with weakly dissipative plasmas, viscosity and magnetic diffusivity are essential only in the dissipative layer. Far away from the dissipative layer the amplitudes of perturbations are small. These two facts enable us to consider that outside the dissipative layer, the plasma motion is described by the ideal linear MHD equations. Another assumption is related to the behaviour of the equilibrium quantities. We suppose that these variables change only slightly across the dissipative layer and they can be approximated by the first non-vanishing term in their Taylor series expansion with respect to the inhomogeneity coordinate, $r$. Similar to the linear theory, we assume that these expansions provide suitable approximations for the equilibrium quantities in the region embracing the ideal resonant position that is much wider than the dissipative layer. This implies that there are two overlapping regions to both sides of the dissipative layer where both the outer (the solution of the linear ideal MHD) and inner solutions (the solution of the nonlinear dissipative MHD) are valid. The two solutions have to coincide in the overlapping regions which provides a matching condition.

Let us proceed to the derivation of the governing equations. In the first step we obtain the solution outside the dissipative layer. As it was pointed out, in this region, the wave dynamics is described by ideal linear MHD. The system of linearized ideal MHD equations can be reduced to a system of two coupled first order PDEs for the radial component of the velocity, $u$, and the Eulerian perturbation of the total pressure, $P$

$D \frac{\partial(u r)}{\partial r}=C_{1} u r+\omega C_{2} r \frac{\partial P}{\partial \theta}$,

and

$\omega r D \frac{\partial^{2} P}{\partial r \partial \theta}=C_{3} u r-\omega r C_{1} \frac{\partial P}{\partial \theta}$, where

$$
\begin{gathered}
D=\rho_{0} D_{A} D_{C}, \quad D_{A}=\omega^{2}-\omega_{A}^{2}, \\
D_{C}=\left(c_{S}^{2}+v_{A}^{2}\right)\left(\omega^{2}-\omega_{T}^{2}\right),
\end{gathered}
$$

and

$$
\begin{gathered}
C_{1}=2 \omega^{4} \frac{B_{0 \varphi}^{2}}{\mu r}-2 \frac{m f_{B} B_{0 \varphi}}{\mu r^{2}} D_{C}, \\
C_{2}=\omega^{4}-\left(\frac{m^{2}}{r^{2}}+k^{2}\right) D_{C}, \\
C_{3}=D\left[\rho D_{A} \frac{\partial^{2}}{\partial \theta^{2}}+\frac{2 B_{0 \varphi}}{\mu} \frac{d}{d r}\left(\frac{B_{0 \varphi}}{r}\right)\right]- \\
-4 \omega^{4}\left(\frac{B_{0 \varphi}^{2}}{\mu r}\right)^{2}+\frac{4 \rho_{0} D_{C} \omega_{A}^{2}}{\mu r^{2}} B_{0 \varphi}^{2} .
\end{gathered}
$$

All other variables can be calculated in terms of these two variables. By eliminating the pressure from Eqs. (11)-(12), we obtain a second order differential equation for the radial component of the velocity

$$
\frac{\partial}{\partial r}\left[f(r) \frac{\partial(u r)}{\partial r}\right]-g(r) \frac{\partial^{2}(u r)}{\partial \theta^{2}}=0,
$$

where

$$
f(r)=\frac{D}{r C_{2}}, \quad g(r)=\frac{\partial}{\partial r}\left(\frac{C_{1}}{r C_{2}}\right)-\frac{1}{r D}\left(\frac{C_{3}}{\omega}-\frac{C_{1}^{2}}{C_{2}}\right)
$$

We are interested in the solution of the system (11)-(12) in the vicinity of the slow wave resonant point, i.e. at $r=r_{c}$ determined by the condition $\omega=\omega_{T}\left(r=r_{c}\right)$, where $\omega$ is the driver frequency. The waves which satisfy this condition are called resonant waves. Let us introduce a new radial variable, $s$, defined by

$s=r-r_{c}$

The $s=0$ point (the resonant position) is a regular singular point of the system (11)-(12) and therefore we look for solutions in the form of Fröbenius expansions around the resonant position $s=0$. The solutions take the form

$P=P_{1}(\theta)+P_{2}(\theta) s \ln |s|+P_{3}(\theta) s+\ldots$,

and

$u=u_{1}(\theta) \ln |s|+u_{2}(\theta)+u_{3}(\theta) s \ln |s|+\ldots$.

Here dots denote terms that are of higher order with respect to $s$. In general, the coefficient functions of $\theta$ in Eqs. (18)(19) are different for $s<0$ and $s>0$.

Using the relations found for the other variables, we eventually find that the perpendicular components of the velocity and magnetic field perturbation behave like the total pressure perturbation, so they are regular at $s=0$. The other quantities are singular. The quantities $u$ and $b_{r}$ behave like $\ln |s|$, while the quantities $v_{\|}, b_{\|}, p, \rho$ have an $s^{-1}$ singularity. These latter quantities are called large variables. 
The outer solution is the so-called large-scale mode, because the transverse scale of this motion is of the order of the physical model (e.g. the diameter of a coronal loop). However, near to the resonant position, in the inner region, the character of the solution changes and the assumption of ideal MHD is not valid. In this region we must have the dissipative, small-scale inner solution.

The magnitude of the dissipation is given by the total Reynolds number defined in the Introduction where the magnetic and viscous Reynolds numbers have the properties

$R_{m} \sim \frac{1}{\bar{\eta}}, \quad R_{v} \sim \frac{1}{\bar{v}}$

If $f$ is a large variable, then a typical representative of the large nonlinear terms is of the form $f \partial f / \partial z$. The typical representative of large dissipative terms is of the form $\partial^{2} f / \partial s^{2}$ multiplied by one of the dissipative coefficients ( $v$ or $\eta$ ). Since inside the dissipative layer the large variables are of the order of $f \sim \epsilon R^{1 / 3}$ and $\partial / \partial z \sim l_{\text {inh }}^{-1}, \partial / \partial s \sim l_{\text {dis }}^{-1}$ the ratio of the nonlinear terms to the dissipative terms is estimated to be

$$
\frac{f \partial f / \partial z}{\bar{v} \partial^{2} f / \partial s^{2}} \sim \epsilon R^{2 / 3}
$$

and $\epsilon R^{2 / 3}$ emerges as the nonlinearity parameter.

If $\epsilon R^{2 / 3} \ll 1$ is satisfied, then linear theory gives an adequate description of the motions in the dissipative layer. However, for combinations of $\epsilon$ and $R$ such that $\epsilon R^{2 / 3} \sim 1$, and definitely for $\epsilon R^{2 / 3} \gg 1$, nonlinearity has to be taken into account when studying resonant waves in the dissipative layer. Linear theory is a valid approximation for the description of the wave dynamics in the dissipative layer if the dissipative terms are much larger than the nonlinear terms and so linear theory can be used if $\epsilon \ll R^{-2 / 3}$.

In linear theory, terms describing dissipation in the MHD equations are retained inside the dissipative layer to remove the singularity. The nonlinear terms have to be taken into account in the dissipative layer if they are of the same order or larger than the dissipative terms, so that $\epsilon R^{2 / 3} \sim 1$, i.e. $R \sim \epsilon^{-3 / 2}$. Linear studies of velocity scaling laws $(v \sim$ $R^{1 / 3}$ ) indicate that the predicted velocities in the dissipative layer are several orders of magnitude larger than the observed nonthermal velocities if the linear results are scaled to match the observed heating rate. This lead to the suggestion that nonlinear effects, important in the dissipative layer, might enhance dissipation and alter the linear velocity scaling law. Therefore, according to Eq. (20), we can scale the dissipative coefficients as

$\bar{v}=\epsilon^{3 / 2} \nu, \quad \bar{\eta}=\epsilon^{3 / 2} \eta$.

In order to obtain the solutions in the internal region, we introduce a new stretching variable in the system of equations. The thickness of the dissipative layer is of the order of $l_{i n h} R^{-1 / 3}$ and since we assume that in the dissipative layer the nonlinear and dissipative terms are of the same order $\left(R \sim \epsilon^{-3 / 2}\right)$, the new variable is $\tau=\epsilon^{-1 / 2} s$, i.e. $r=\tau^{\prime}=r_{c}+\epsilon^{1 / 2} \tau$.
The system of equations obtained in this way contain $\epsilon^{1 / 2}$, so we use this quantity as an expansion parameter. To find the expansion form for the variables in the dissipative layer, we have to analyze the form of the outer expansions given by Eqs. (18)-(19). Since the quantities $v_{\perp}, b_{\perp}$ and $P$ are regular in the vicinity of $s=0$, their amplitudes in the dissipative layer have to be the same as the amplitude outside this layer. Therefore, the expansion of this quantity is

$f=\epsilon f^{(1)}+\epsilon^{3 / 2} f^{(2)}+\ldots$

It is easy to verify that the amplitudes of the large variables in the dissipative layer is of the order of $\epsilon^{1 / 2}$, so we can write the expansion for $v_{\|}, b_{\|}, p$ and $\rho$ in the form

$g=\epsilon^{1 / 2} g^{(1)}+\epsilon g^{(2)}+\ldots$

The quantities $u$ and $b_{r}$ have an $\ln |s|$ behaviour near to the resonant position $s=0$, so they are of the order of $\epsilon \ln \epsilon$ in the dissipative layer, which means that we have to start the expansions of these quantities with this term. It was shown by Ruderman et al. (1997a) that expansions (23) and (24) also contain terms proportional to $\epsilon^{3 / 2} \ln \epsilon$ and $\epsilon \ln \epsilon$. However, according to the results found by Ballai et al. (1998a) in the simplified version of the method of asymptotic expansion we use the fact that $\ln |\epsilon| \ll \epsilon^{-\kappa}$ for any positive $\kappa$ and $\epsilon \rightarrow+0$, and we consider $\ln \epsilon$ to be of the order of one in the dissipative layer. This enables us to write the expansion for $u$ and $b_{r}$ in the form of Eq. (23).

In the first order approximation we obtain a system of homogeneous linear equations for the variables with the superscript ' 11 '. The quantities we need to express with the variables of the second order can be written with the aid of $P^{(1)}$, $u^{(1)}$ and $v_{\|}^{(1)}$ as

$b_{\|}^{(1)}=-\frac{\omega f_{B}}{\omega_{A}^{2}} v_{\|}^{(1)}, \quad b_{r}^{(1)}=-\frac{f_{B}}{\omega} u^{(1)}$,

$p^{(1)}=\frac{B_{0} \omega \rho_{0}}{f_{B}} v_{\|}^{(1)}, \quad \rho^{(1)}=\frac{B_{0} \omega \rho_{0}}{c_{S}^{2} f_{B}} v_{\|}^{(1)}$.

The radial component of the momentum equation connects the derivative of the total pressure perturbation and the parallel component of the velocity

$\frac{\partial P^{(1)}}{\partial \tau}=\frac{2 B_{0 \varphi}^{2} \omega \rho_{0}}{B_{0} f_{B} r_{c}} v_{\|}^{(1)}$,

and the equation that relates the normal and parallel component of velocity is

$\frac{\partial u^{(1)}}{\partial \tau}+\frac{\omega^{2}}{\omega_{A}^{2}} \frac{f_{B}}{B_{0}} \frac{\partial v_{\|}^{(1)}}{\partial \theta}=0$,

where the equilibrium quantities are evaluated at the resonant point. We can see that for $B_{0 \varphi}=0$ we recover the results for the straight equilibrium magnetic field found by Ballai et al. (2000). By combining the two last equations, we obtain a conservation law similar to the linear theory, i.e.

$\frac{d P^{(1)}}{d \theta}+\frac{2 B_{0 \varphi}^{2}}{\omega \mu r_{c}} u^{(1)}=\mathcal{C}^{(1)}(\theta)$, 
where $\mathcal{C}^{(1)}(\theta)$ is the first term in a series expansion of the coefficient $\mathcal{C}(\theta)$, i.e. $\mathcal{C}(\theta) \simeq \epsilon \mathcal{C}^{(1)}(\theta)$. In the second order approximation, we obtain a system which can be derived from the left-hand sides of the corresponding equations of the first order approximation by substituting variables with superscripts ' 2 ' for the corresponding variables with superscripts ' 11 '. Since the set of first order approximation equations possess a non-trivial solution, the second order equations are compatible only if the first order terms satisfy a compatibility condition. Eventually, we obtain a nonlinear partial differential equation which describes the dynamics of resonant slow MHD waves in the dissipative layer

$$
\begin{aligned}
& \Delta \tau \frac{\partial v_{\|}^{(1)}}{\partial \theta}-\frac{\omega^{3} B_{0}\left[(\gamma+1) v_{A}^{2}+3 c_{S}^{2}\right]}{f_{B} c_{S}^{2}\left(c_{S}^{2}+v_{A}^{2}\right)} v_{\|}^{(1)} \frac{\partial v_{\|}^{(1)}}{\partial \theta}+ \\
& \quad+\omega\left(v+\frac{\omega_{T}^{2}}{\omega_{A}^{2}} \eta\right) \frac{\partial^{2} v_{\|}^{(1)}}{\partial \tau^{2}}=\frac{\omega^{3} B_{0}}{\rho_{0} v_{A}^{2} f_{B}} \mathcal{C}^{(1)}(\theta),
\end{aligned}
$$

where

$\Delta=-\frac{d \omega_{T}^{2}}{d s}(s=0)$.

Similar to the $B_{0 \varphi}=0$ case investigated by Ballai et al. (2000), the driving term is the quantity which does not change across the dissipative layer. Equation (30) is the nonlinear governing equation for the parallel velocity in the dissipative layer for slow resonant waves. The second term in the left-hand side is the nonlinear term and the third term is the dissipative term. The term in the right-hand side of Eq. (30) is determined by the solution outside the dissipative layer and its form can be prescribed.

The resonant surface $s=0$ can be considered as a surface of discontinuity when solving the system of equations that govern the plasma motion outside the dissipative layer, i.e. we have to calculate the jumps in the physical parameters. The solution of Eq. (30) has to vanish at infinity and has to satisfy the condition that its mean value over a period with respect to $\theta$ is zero.

The jump of a function $f(r)$ across the dissipative layer is defined by

$$
[f]=\lim _{s \rightarrow+0}\{f(s)-f(-s)\} .
$$

Let us introduce new dimensionless variables

$\sigma=\delta_{c}^{-1} \epsilon^{1 / 2} \tau, \quad q=\epsilon^{1 / 2} \frac{\omega \delta_{c}}{v_{A c}^{2}} v_{\|}^{(1)}$,

where $\delta_{c}$ measures the thickness of the dissipative layer in isotropic plasmas and is defined by the condition that the first and the third terms in the left-hand side of the governing Eq. (30) are of the same order. Its form is given by

$\delta_{c}=\left[\frac{\omega}{|\Delta|}\left(\bar{v}+\frac{\omega_{T c}^{2}}{\omega_{A c}^{2}} \bar{\eta}\right)\right]^{1 / 3}$.

Let $r_{0}$ be the characteristic width of the overlap regions to the left and right of the dissipative layer, where both the linear ideal and the nonlinear and dissipative MHD equations with coefficients approximated by the first non-zero terms of the Taylor expansions are valid. The main property of the variable $\sigma$ introduced in Eq. (32) is that $\sigma=\mathcal{O}(1)$ in the dissipative layer, while $|r| \rightarrow r_{0}$ corresponds to $|\sigma| \rightarrow \infty$. In agreement to the matching procedure, the inner and the outer solutions have to be identical in the overlapping regions. This condition provides us with another definition of the jump in the function $f(r)$ across the dissipative layer

$[f]=\lim _{\sigma \rightarrow \infty}\{f(\sigma)-f(-\sigma)\}$.

In the new variables, the governing equation becomes

$\sigma \frac{\partial q}{\partial \theta}+\Lambda q \frac{\partial q}{\partial \theta}-\frac{\partial^{2} q}{\partial \sigma^{2}}=-\frac{\omega^{4} B_{0}}{v_{A}^{4} f_{B} \rho_{0}|\Delta|} \mathcal{C}(\theta)$,

where $\Lambda$ can be expressed by the equilibrium quantities at the resonant position. Here, the approximations $u \approx \epsilon u^{(1)}$ $C(\theta) \approx \epsilon C(\theta)^{(1)}$ are used outside the dissipative layer.

In order to derive the two connection formulae, we introduce the new variables in the relations (27) and (28) and using Eq. (34) we finally obtain

$[P]=\frac{2 B_{0 \varphi}^{2} B_{0}}{\mu r_{c} f_{B}} \mathcal{P} \int_{-\infty}^{\infty} q d \sigma$,

and

$[u]=-\frac{\omega f_{B} v_{A}^{2}}{\omega_{A}^{2} B_{0}} \mathcal{P} \int_{-\infty}^{\infty} \frac{\partial q}{\partial \theta} d \sigma$,

where we used the symbol of Cauchy principal part $\mathcal{P}$ since the integrals are divergent at infinity. We can see that for a magnetic field with straight lines $\left(B_{0 \varphi}=0\right)$, the jump in the total pressure becomes zero, i.e. this quantity is conserved across the singularity. These two equations are the nonlinear analog of the connection formulae for the radial component of the velocity and total pressure perturbation obtained in linear theory by Sakurai et al. (1991). However, in contrast to the linear theory where the jump conditions were given as a function of equilibrium quantities and the perturbation of the total pressure, the nonlinear connection formulae are given in terms of an integral of an unknown function, $q$. Therefore, we have to solve simultaneously the system (11)-(12) describing the wave motion outside the dissipative layer and Eq. (35), which describe the wave dynamics inside the dissipative layer, with Eqs. (36)-(37) providing boundary conditions for the problem.

\section{Generation of mean flow}

One of the most interesting effects of nonlinearity in the dissipative layer is the generation of a mean flow outside the dissipative layer due to absorption of wave momentum. For the sake of simplicity, we consider this problem in the untwisted case, i.e. $B_{0 \varphi}=0$.

In linear theory all perturbed quantities are harmonic functions of $\theta$ and their mean values over a period vanishes. In 
nonlinear theory, however, the mean value of perturbations can have a non-zero value due to the interaction of the different harmonics. This interaction generates a mean flow outside the dissipative layer. The mean value of a quantity $f(\theta)$ over a period $L$ is defined by

$\langle f\rangle=\frac{1}{L} \int_{0}^{L} f(\theta) d \theta$.

Let us divide the $\varphi$ - and $z$-components of velocity into mean and oscillatory parts, as follows:

$v=U_{\varphi}+\tilde{v}, \quad w=U_{z}+\tilde{w}, \quad\langle v\rangle=U_{\varphi}, \quad\langle w\rangle=U_{z}$,

where quantities with a tilde denote the oscillatory part of the velocities. The quantities $U_{\varphi}$ and $U_{z}$, respectively, describe the mean flow components.

This flow is in the plane parallel to the dissipative layer, parallel and perpendicular to the magnetic field lines. Its amplitude is determined by the balance between the forces created by resonant absorption and shear viscosity. After long and cumbersome calculations (for details see, e.g. Ruderman et al., 1997a; Ballai et al., 2000) we see that the components of the generated flow are continuous functions of $r$, but their vorticity have a jump across the dissipative layer given by

$$
\begin{gathered}
{\left[V_{\varphi}\right]=\left[\frac{d U_{\perp}}{d s}\right]=-\frac{m}{r_{c} \omega}\left(1+\frac{\omega_{T c}^{2}}{\omega_{A c}^{2}} P_{m}^{-1}\right) \times} \\
\int_{-\infty}^{\infty}\left\langle\left(\frac{\partial \tilde{w}}{\partial s}\right)^{2}\right\rangle d s \\
{\left[V_{\|}\right]=\left[\frac{d U_{z}}{d s}\right]=-\frac{\omega}{k v_{A}^{2}}\left(1+P_{m}^{-1}\right) \int_{-\infty}^{\infty}\left\langle\left(\frac{\partial \tilde{v}}{\partial s}\right)^{2}\right\rangle d s}
\end{gathered}
$$

where $P_{m}=v / \eta$ is the magnetic Prandtl number. Keeping in mind solar applications, the value of this flow may reach about a few $\mathrm{km} / \mathrm{s}$. The existence of this mean flow was predicted numerically by Ofman and Davila (1995). This turbulent flow might be subject to Kelvin-Helmholtz instability which could destroy the dissipative layer, and the process of resonant absorption ceases to exist. At the same time, the turbulent flow outside the dissipative layer may enhance locally the dissipative coefficients which could lead to a better energy transfer.

\section{Conclusions}

The aim of the present paper is to study the nonlinear behaviour of resonant slow MHD waves in twisted magnetic flux tubes analytically. The applied scaling method divides the domain into two regions, where the wave behaviour and dynamics are governed by different sets of equations.

In the outer domain, i.e. outside the dissipative layer, the wave motion is described by the ideal linear MHD equations which can be reduced to a pair of coupled first order PDE's derived for the radial component of the velocity and the total pressure perturbation.
In the inner domain, i.e. in the dissipative layer, the wave dynamics is governed by an inhomogeneous nonlinear PDE derived for the parallel component of the velocity, where the inhomogeneous part originates from the driving term. Since the dissipative layer embracing the resonant surface is very narrow, it can be considered as a surface of discontinuity when solving the governing PDE's outside the dissipative layer. The connection formulae obtained for the total pressure perturbation and the radial component of the velocity provides the jumps in these quantities across the dissipative layer, therefore providing boundary conditions at the resonant surface of discontinuity. In contrast to the case with a straight equilibrium magnetic field, the Eulerian perturbation of the total pressure is no longer a conserved quantity. Instead, we found that the conserved quantity is a combination of the total pressure and the radial component of the velocity, as it was found in linear theory.

The nonlinear interaction between harmonics generates a mean flow outside the dissipative layer. Analytical expressions are found for the mean flow which are piecewise linear continuous functions of the radial coordinate, $r$. However, the vorticity of these shear flows exhibits a jump across the dissipative layer. For a strong flow, this process can be subject to Kelvin-Helmholtz instability which could destroy the dissipative layer.

Acknowledgements. R. Erdélyi acknowledges M. Kéray for patient encouragement. RE and IB also aknowledge the financial support obtained from the NSF Hungary (OTKA, ref nr. TO32462). The authors also thank M.S. Ruderman for valuable discussions and suggestions. We also thank the unknown Referees for their comments.

\section{References}

Ballai, I., Erdélyi, R., and Ruderman, M. S.: Interaction of sound waves with slow dissipative layers in anisotropic plasmas in the approximation of weak nonlinearity, Phys. Plasmas, 5, 2264, 1998a.

Ballai, I., Ruderman, M. S., and Erdélyi, R.: Nonlinear theory of slow dissipative layers in anisotropic plasmas, Phys. Plasmas, 5, 252, 1998b.

Ballai, I., Erdélyi, R., and Goossens, M.: Nonlinear theory of non-axisymmetric resonant slow waves in straight magnetic flux tubes, J. Plasma Phys., 64, 235, 2000.

Bray, R. J., Cram, L. E., and Durrant, C. J.: Plasma Loops in the Solar Corona, Cambridge University Press, Cambridge, 1991.

Belien, A. J. C., Poedts, S., and Goedbloed, J. P.: Continuous magnetohydrodynamic spectra of two-dimensional coronal magnetic flux tubes, Astron. Astrophys., 322, 995, 1997.

Davila, J. M.: Heating of the solar corona by the resonant absorption of Alfvén waves, Astrophys. J., 317, 514, 1987.

de Bruyne, P., Parhi, S., and Goossens, M.: Numerical simulations of driven MHD waves in twisted coronal loops, Astrophys. Lett. Comm., 34, 163, 1996.

Edwin, P. M. and Roberts, R.: The Benjamin-Ono-Burgers equation: An application in solar physics, Wave Motion, 8, 151, 1986.

Erdélyi, R. and Goossens, M.: Viscous computations of resonant absorption of MHD waves in flux tubes by FEM, Astrophys. Space Sci, 213 273, 1994. 
Erdélyi, R.: Analytical solutions for cusp resonance in dissipative MHD, Solar Phys., 171, 49, 1997.

Erdélyi, R. and Ballai, I.: Resonant absorption of nonlinear slow MHD waves in isotropic steady plasmas, Solar Phys., 186, 67, 1999.

Erdélyi, R., Ballai, I., and Goossens, M.: Nonlinear resonant absorption of fast magnetoacoustic waves due to coupling into slow continua in the solar atmosphere, Astron. Astrophys., 368, 662, 2001.

Golub, L. and Pasachoff, J. M.: The Solar Corona, Cambridge University Press, Cambridge, 1997.

Goossens, M.: Magnetohydrodynamic waves and wave heating in nonuniform plasmas, in: Advances in Solar System Magnetohydrodynamics, (Eds) Priest, E. R. and Hood, A. W. (Cambridge Univ. Press, Cambridge), 135, 1991.

Goossens, M. and Poedts, S.: Linear resistive magnetohydrodynamic computations of resonant absorption of acoustic oscillations in sunspots, Astrophys. J., 384, 148, 1992.

Goossens, M. and Hollweg, J. V.: Resonant behaviour of MHD waves on magnetic flux tubes. IV - Total resonant absorption and MHD radiating eigenmodes, Solar Phys., 145, 19, 1993.

Goossens, M., Ruderman, M. S., and Hollweg, J. V.: Dissipative MHD solutions for resonant Alfvén waves in 1-dimensional magnetic flux tubes, Solar Phys., 157, 75, 1995.

Hollweg, J. V.: Resonance absorption of solar p-modes by sunspots, Astrophys. J., 335, 1005, 1988.

Hollweg, J. V.: Alfvén waves, in: Mechanism of Chromospheric and Coronal Heating, (Eds) Ulmschneider, P., Priest, E. R. and Rosner, R., Springer-Verlag, Berlin, 423, 1991.

Ionson, J. A.: Resonant absorption of Alfvénic surface waves and the heating of solar coronal loops, Astrophys. J., 226, 650, 1978.

Kuperus, M., Ionson, J. A., and Spicer, D.: On the theory of coronal heating mechanisms, Ann. Rev. Astron. Astrophys., 19, 7, 1981.

Litwin, C. and Rosner, R.: Alfvén wave transmission and heating of solar coronal loops, Astrophys. J., 499, 945, 1998.

Lou, Y. Q.: Viscous magnetohydrodynamic modes and p-mode absorption by sunspots, Astrophys. J., 350, 452, 1990.

Narain, U. and Ulmschneider, P.: Chromospheric and coronal heating mechanisms, Space Sci. Rev., 54, 377, 1990.

Narain, U. and Ulmschneider, P.: Chromospheric and coronal heating mechanisms II, Space Sci. Rev., 75, 453, 1996.

Nayfeh, A. H.: Introduction to Perturbation Techniques, WileyInterscience, New York, 1981.

Ofman, L. and Davila, J. M.: Nonlinear resonant absorption of Alfvén waves in three dimensions, scaling laws, and coronal heating, J. Geophys. Res., 100, 23 427, 1995.

Ruderman, M. S., Goossens, M., and Hollweg, J. V.: Nonlinear theory of resonant slow waves in dissipative layers, Phys. Plasmas, 4, 75, 1997a.

Ruderman, M. S., Hollweg, J. V., and Goossens, M.: Nonlinear theory of the interaction of sound waves with an inhomogeneous magnetized plasma in the resonant slow wave layer, Phys. Plasmas, 4, 92, 1997b.

Ruderman, M. S.: Interaction of sound waves with an inhomogeneous magnetized plasma in a strongly nonlinear resonant slowwave layer, J. Plasma Phys., 63, 43, 2000.

Sakai, J. I., Minamizuka, R., Kawata, T. et al.: Nonlinear torsional and compressional waves in a magnetic flux tube with electric current near the quiet solar photospheric network, Astrophys. J., 550, 1075, 2001.

Sakurai, T., Goossens, M., and Hollweg, J. V.: Resonant behaviour of MHD waves on magnetic flux tubes, I-Connection formulae at the resonant surfaces, Solar Phys., 133, 227, 1991. 